\title{
Introduction to the Special Issue on the COVID-19 Emergency Transition to Remote Learning
}

\author{
Shanna Smith Jaggars \\ The Ohio State University
}

The COVID-19 pandemic began in December 2019, and within three months had spread across Europe, the United States, and almost every other nation in the world. To help contain the deadly virus, countries across the globe closed the doors of K-12 schools and colleges. Students residing on university campuses were sent home. School administrators and instructors scrambled to devise plans for teaching and supporting their students from their own homes. Depending on their local context, their experience and training, and their students' desires and needs, some teachers turned to teaching by correspondence, some to asynchronous online learning, some to synchronous remote class sessions, and some to a unique mix of these elements. Administrators, teachers, and students may have expected these emergency distance education practices to be temporary - a few weeks at most - but many were required to continue with remote teaching through the spring of 2021. More than a year after it began, the pandemic may at last be coming under control as vaccines are distributed across the world, and teachers and students are cautiously looking forward to a return to "normal" schooling conditions in the autumn of 2021. However, lessons from this extended period of universal remote learning will influence student instruction and support practices for many years to come, for both online and in-person courses and programs.

The articles in this Special Issue provide a rich portrait of the teaching and learning challenges which characterized the initial emergency transition in Spring 2020, and detail the approaches of administrators and teachers as they attempted to overcome those challenges. Along the way, these studies provide lessons in terms of how to better prepare for future public emergencies, as well as how to improve student success more generally, in both online and inperson settings. Throughout the issue, readers will also see a multitude of challenges related to the "digital divide" - or the fact that students have unequal access to reliable high-speed Internet and other academic technologies, due to underlying inequalities in household income and regional infrastructure.

The Special Issue is organized into three sections: the first includes a selection of fulllength manuscripts focused on teaching, learning, and student support challenges in the U.S.; the second provides a series of brief case studies which delve into how specific courses or programs managed the transition; and the third provides a set of international perspectives. 


\section{Section I: Investigating Teaching, Learning, and Student Supports in the U.S.}

This section opens with a broad overview of how U.S. college students experienced the emergency transition to online learning, and then delves into how institutions deployed supports to students, and how K-12 and college instructors worked to adapt their instruction in the remote context.

The opening article, "Teaching and Learning in the Time of COVID: The Student Perspective" by Barbara Means and Julie Neisler, provides a broad overview of the U.S. college student perspective on the emergency transition. The article describes findings from the Survey of Student Perceptions of Remote Teaching and Learning, which was administered to a random national sample of 1,008 U.S. undergraduates in spring 2020. Students struggled with the digital divide, academic motivation, and other challenges, and these challenges were exacerbated among students of color and those from lower-income households. However, when instructors leveraged practices which the literature has consistently recommended for effective online instruction, their students were shielded against some of the negative impacts of the emergency transition.

The next two articles in this section explore how U.S. colleges supported their students through the emergency transition using tools, services, instructor training, and other institutionwide practices. In "Remote Student Support During COVID-19: Perspectives of Chief Online Officers in Higher Education," Bettyjo Bouchey, Erin Gratz, and Shelley Kurland report on a set of 31 interviews with chief online officers at colleges across the country. Results suggest that support services can be effectively delivered online, and that these remote supports can be valuable for all students (not just those enrolled in online degree programs). Moreover, institutions which invested in online programs and supports prior to COVID-19 were more nimble and efficient in their pivot to emergency remote operations. Thus the authors recommend that institutions move away from a "siloed" model in which a small group of staff work exclusively to support fully online students, and toward an "integrated" model in which knowledge and skills regarding online students, courses, and supports are spread across the entire institution. This message is broadly consistent with the findings of the next article, "COVID-19 and Community College Instructional Responses," by Cassandra Hart, Di Xu, Michael Hill, and Emily Alonso, which gathered information from 45 distance education leaders across the California community college system. Hart and her colleagues find that some community colleges were better-positioned than others for the unexpected crisis, and this positioning was at least partially related to membership in an interinstitutional collaborative focused on online learning (the California Virtual Campus - Online Education Initiative). Colleges with a stronger foundation in online programs and supports were able to train students in a greater variety of online learning skills and offer a greater number of approaches to training faculty. The authors also found that all campuses made efforts to provide adequate Internet access and computing hardware to needy students. However, respondents estimated that perhaps one-third of California community college students would still face technology barriers to remote learning in the autumn of 2020.

The next two articles in this section provide a large-scale picture regarding how U.S. college instructors adapted their teaching during the emergency transition. The first article, "A Pandemic of Busywork: Increased Online Coursework Following the Transition to Remote Instruction is Associated with Reduced Academic Achievement" by Benjamin Motz, Joshua Quick, Julie Wernert, and Tonya Miles, suggests that instructional quality suffered during the emergency transition. Leveraging a survey of 4,636 students at a large multi-campus university in the spring of 2020, the authors linked survey responses to student activity logs and course 
performance data. Results suggest that many instructors replaced face-to-face time with "busywork" assignments, and as a result, their students spent more time and effort on coursework without improving their learning or performance. Why might instructors take such a counterproductive step? The next article provides some potential clues. In "Profiles of Instructor Responses to Emergency Distance Learning," Teomara Rutherford, Sarah Karamarkovich, Di Xu, Tamara Tate, Brian Sato, Rachel Baker, and Mark Warschauer surveyed 137 faculty at a large U.S. university in the spring of 2020, focusing on respondents' usage of and confidence in their ability to implement a set of evidence-based teaching practices, in both their emergency remote course and in the previous in-person instance of that course. When moving into the remote emergency context, instructors downshifted in terms of their confidence and usage of these practices. Based on their data, the authors provide two potential reasons why instructors might downshift. First, perhaps instructors who were skilled facilitators of in-person classrooms were unsure how to implement these student-centered practices in a remote context, and thus fell back on more instructor-centered practices. Second, they speculate that some instructors purposefully reduced collaboration and peer work as a sign of caring for their students, in order to accommodate the other life challenges students were experiencing during a pandemic.

Building on the theme of caring, the next three articles explore how K-12 and college instructors expressed care for their students during the emergency transition to remote learning. In “A Light in Students' Lives: K-12 Teachers' Experiences (Re)Building Caring Relationships During Remote Learning," Karyn Miller gathered qualitative data from twenty K-12 teachers who were enrolled in a graduate course. Teachers provided data through reflective journals and discussion forums both before and after the emergency transition, allowing the author to trace how teachers' connections to their K-12 students shifted as school moved online. At the outset of the spring 2020 semester, teachers were already experiencing normal and ongoing challenges regarding relationship-building with their students; but in the face of the emergency transition, these teachers re-prioritized relationship-building. Miller details the perspectives and behaviors these teachers implemented in order to build strong relationships, including establishing a positive tone, insisting on high expectations, responding to students' non-academic needs, and encouraging peer interaction. The next study complements this teacher perspective with a student perspective on instructor caring and connectedness. In "Instructor Social Presence and Connectedness in a Quick Shift from Face-to-Face to Online Instruction," Sheri Conklin and Amy Garrett Dikkers surveyed 432 undergraduate and graduate students at a U.S. university in spring 2020, and asked them to focus on a class that helped them stay connected with their instructor, content, and peers. Among other findings, the results suggest that students feel more connected when instructors leverage video (including synchronous video meetings and instructor-created asynchronous video content), use a conversational tone, incorporate empathic messages into course communications, and provide timely feedback. Such behaviors are important to the success of all students, but as the next article shows, they may also be particularly important for student equity. In "Using an Ethos of Care to Bridge the Digital Divide: Exploring Faculty Narratives During a Global Pandemic," Kari Goin Kono and Sonja Taylor interviewed 20 faculty at a large U.S. university, with a focus on equitable teaching practices. A key contribution of this study is its explicit invocation of culturally-responsive pedagogy, an equity-building approach to instruction which is becoming more well-known in K-12 education but is largely absent from studies of online learning in higher education. This study draws clear connections between responsive pedagogy and the bridging of the digital divide, and helps establish the need for culturally-responsive pedagogy to play a more prominent role in the study and practice of online learning. 
The final article in this section looks to the future of online teaching in higher education: graduate students. In "Preparing Doctoral Students to Teach in an Increasingly Virtual World: A Response to COVID-19 and Beyond," Robbie Bishop-Monroe, Brandon Di Paulo Harrison, Margaret Knight, Cynthia Corritore, Brian Rybarczyk, and Anne Stewart York investigate whether and how graduate students were prepared for emergency remote teaching in spring 2020, and more broadly, for online teaching in their future careers as academics. The authors used multiple data sources across two universities to address these questions, including a survey of 63 current or recent doctoral students, interviews with 12 department chairs and deans, and qualitative data from 18 participants in a four-week "bootcamp" on online teaching. Results suggest that doctoral students believe online teaching is important, and that current and recent graduate students benefit from training on the topic; however, even in the face of the COVID-19 pandemic, deans and department chairs downplayed the importance of online teaching and did not see a clear necessity to provide doctoral students with training on the topic.

\section{Section II: Brief Case Studies on the Pivot to Emergency Remote Teaching}

In response to the Call for Submissions for this special issue, the editors received a large volume of submissions which focused on how a specific course or academic program managed the transition to emergency remote learning during the spring of 2020. In general, these submissions documented serious challenges, but also recounted a successful experience from which other instructors might learn. Of course, there may be a multitude of unsubmitted and never-to-bepublished examples of poorly-managed emergency transitions. As such, this body of case studies should not be taken as evidence that the emergency transition was a consistent story of triumph over adversity. Yet from these examples of success, the field can take lessons in terms of how to more consistently ensure that online courses provide high-quality learning experiences, even in the context of a public emergency.

Most studies in this section focus on hands-on or clinical experiences, which in the past have been challenging to provide online. In order to include a variety of different studies given the limitations of space, studies in this section are shorter than a typical manuscript, with an abbreviated literature review. To provide maximum guidance for practice, authors focus on providing a short overview of their particular context, a detailed description of the practices they implemented to support student learning, and descriptive evidence regarding the extent to which student satisfaction or learning remained consistent or changed across the spring 2020 semester.

The first two studies in this section focus on extracurricular activities, which abruptly disappeared for many students midway through the spring 2020 semester. In "Without Crossing a Border: Exploring the Impact of Shifting Study Abroad Online on Students' Learning and Intercultural Competence Development during the COVID-19 Pandemic," Yingjie Liu and Thomas Shirley discuss how virtual reality technologies helped students in the U.S., Germany, Brazil, and India create and share cultural exchange virtual tours, while supporting their development of intercultural competence. In "Motivating Students to Learn AI Through Social Networking Sites: A Case Study in Hong Kong," Tsz Kit Ng and Kai Wa Chu report on a threephase action research process, in which teachers of an extracurricular course on artificial intelligence iteratively improved its delivery based on student feedback. These studies shed light on how extracurricular activities could be delivered remotely even in "normal" times, and thus could potentially provide more access to students who have financial or geographical barriers to participation in traditional in-person extracurriculars. 
The third study in this section may be particularly useful for faculty in STEM fields which require hands-on laboratory experiences. In "Moving Hands-On Mechanical Engineering Experiences Online: Course Redesigns and Student Perspectives," Jaclyn Johnson and Nancy Barr describe how a Mechanical Engineering Practice course sequence was redesigned, and use students' reflective essays and assignment submissions to understand how student perspectives and performance changed with the redesign.

The next three studies focus on remote delivery of clinical experiences. In "Maintaining Academic Performance and Student Satisfaction during the Remote Transition of a Nursing Obstetrics Course to Online Instruction," Elizabeth Riley, Natalie Capps, Nicole Ward, Leslie McCormack, and Judy Staley discuss how live and recorded whiteboard lectures with Socraticstyle questioning, electronic audience response systems, remote simulations, and virtual unfolding case studies served as effective replacements for didactic and clinical learning experiences. The practice of virtual unfolding case studies is examined in more detail in "Using Telehealth to Enhance Pediatric Psychiatric Clinical Simulation: Rising to Meet the COVID-19 Challenge," by Teresa Whited, Kimberly Stickley, Pamela De Gravelles, Taylor Steele, and Beverly English. This study describes a telehealth simulation centered on a pediatric psychiatric patient, and documents students' experiences with the simulation. Moving from mental health to nutrition, in "Adapting a Classroom Simulation Experience to an Online Escape Room in Nutrition Education," Jenifer Ross, Lauri Wright, and Andrea Arikawa describe how they modified a face-to-face "escape room" assignment into a comparable online experience, how students experienced the online assignment, and how instructors plan to modify the assignment to further improve it in the future.

In each of the clinical case studies described above, the authors provided information about how student learning was assessed within the particular course under study. The final case study in this section turns a stronger spotlight on assessment, to interrogate how high-quality student assessment can be implemented in a remote context. In "Moving Assessment Online: Experiences within a School of Pharmacy," Kelsey Morgan, Erin Adams, Teresa Elsobky, Marcia Brackbill, and Amber Darr discuss the benefits, practical logistics, and challenges associated with several forms of remote assessment including a video conferencing technology approach which mimicked pre-pandemic, on-campus proctored exams; open-book, internet access-enabled exams; and virtual observation of clinical skills.

Across all the case studies in this section, the successful transition to remote learning required an intensive investment of institutional and individual instructor resources. For example, in Whited's study, the authors detail the orchestration of personnel required to deliver the simulation to 60 students in four groups across two days:

Students played the part of clinic nurses in pairs and logged-in from their preferred remote individual location. The pediatric APRN faculty and her daughter acted the roles of "Mom" and 15-year-old Jamie. The APRN student assumed the role of pediatric psychiatric clinic APRN. Faculty serving as content experts, chatroom and breakout room monitors, and the facilitator and debriefer, were located in the simulation center and distanced per COVID guidelines. The graduate student role-playing as APRN and the mother-daughter standardized patient actors all participated from their homes. An online learning technology expert from the university's Office of Educational Development agreed to be on stand-by to resolve potential electronic disturbances. The chatroom monitor recorded attendance and observed active engagement of all attendees. 
In addition to new technological tools, training, and infrastructures, the courses profiled in this section required additional preparation time for instructors as well a substantial amount of face-to-face instructional time (albeit deployed virtually online, rather than in an in-person classroom). Accordingly, these courses likely cost as much or more as traditional in-person courses to deploy. Despite their cost, however, some remote elements may be well-worth preserving into the autumn of 2021 and beyond. For example, study abroad offices might consider offering both in-person travel programs and virtual reality experiences, in order to provide opportunities for lower-income students, or those with work or family obligations, who may not have the time or financial resources required for international travel. As another example, Whited's study suggests that nearby in-person clinical settings do not always provide an optimal context for students' learning, and that complementing in-person experiences with virtual "telehealth" approaches can expand students' participation in high-quality clinical experiences.

\section{Section III: International Perspectives}

The COVID-19 pandemic affected education systems across the world, but the extent to which it negatively impacted teaching and learning in a given region may have been dependent on the region's technological infrastructure - including the extent to which reliable high-speed Internet was equally accessible across rural, urban, higher-income, and lower-income areas. The articles in this section provide an inside look at how emergency remote teaching and learning was implemented in parts of the world which had stronger or weaker infrastructures for education and technology.

The first article, "The Forgotten Ones: How Rural Teachers in Mexico are Facing the COVID-19 Pandemic," by Brenda Cecilia Padilla Rodriguez, Alejandro Armellini, and John Traxler, focuses on 75 rural teachers in Mexico, including both K-12 and college teachers. As the authors report, fifteen years ago the Mexican government determined that it was "economically unfeasible" to provide strong education and technology infrastructures for small-population rural areas; accordingly, rural teachers and students had little training or experience with academic technology. When COVID-19 struck, most of the study's respondents reported that they had no broadband Internet access, and that their students did not have adequate Internet, adequate electronic devices, or the skills to learn online. In the face of these challenges, rural teachers drew on their strong resilience and creativity to provide the best possible learning environment for their students, and worked to personalize their teaching to their students' needs. As a result, although $48 \%$ felt student learning was hampered by the changes in their teaching, another $40 \%$ thought the changes to their teaching were positive.

The technology challenges cited by teachers in rural Mexico were echoed by teachers, students, and parents in three developing countries in or near the Middle East. In "The Hidden Shadow of Coronavirus on Education in Developing Countries," Zuheir Khlaif, Soheil Salha, Shahid Fareed, and Hadi Rashed interviewed middle-school students, parents, and teachers in Palestine, Libya, and Afghanistan, and observed the teachers' online courses. In contrast to those in Padilla Rodriguez's study, the teachers in Khlaif's study were more familiar with online teaching, in part because violent disruptions had occasionally necessitated a temporary switch to remote teaching in the past. Even so, teachers and students suffered from electricity outages, weak or unreliable Internet connections, and a lack of technological devices. In addition, female students and their parents in these three countries were particularly concerned about online privacy and security, and many felt uncomfortable participating in synchronous online video sessions due to 
these concerns. In general, teachers' efforts to provide high-quality teaching were not wellsupported by local educational infrastructures in these three countries, which had been weakened by violence and political instability. In contrast, the neighboring country of Jordan, while still economically poor, enjoys a more stable political system and a relatively strong educational infrastructure. In “Jordanian University Students' Views on Emergency Online Learning During COVID-19," Saleh Al-Salman and Ahmad Haider surveyed over 4,000 Jordanian undergraduate students about their experiences with the emergency transition to remote learning. Overall, respondents had mixed feelings about online learning, but students in the sciences were more negative than those in the arts and humanities. Although science students were more likely to have an adequate Internet connection, they were also more frustrated by a mismatch between the demands of their particular discipline and the academic technology available to meet those demands. For example, students in the sciences were much more likely to encounter technical problems while submitting their exams, which Al-Salman and Haider speculate may be due to the necessity of using charts, symbols, and other non-standard assessment approaches in many scientific and technical fields.

The final article in this section, "Teachers' Engagement in and Coping with Emergency Remote Instruction During COVID-19-Induced School Closures: A Multinational Contextual Perspective," by Magdalena Jelinska and Michał B. Paradowski, provides a big-picture view of how the COVID-19 crisis differentially affected educators around the world. Using a survey of nearly 1,500 K-12 and college teachers from 118 countries, the authors identified two clusters of teachers: those who managed the transition relatively well, and those who struggled to cope and engage with remote learning. Their results suggest that teachers were more likely to manage the transition well if they had prior experience with remote instruction, taught in the higher education sector, and taught using real-time synchronous modalities. Interestingly, after controlling for these variables as well as level of coping, teachers in developing countries were more engaged than their similar peers in developed countries. Jelinska and Paradowski speculate that teachers in developing areas may put forth extra effort to support their students, because continuity of education is particularly crucial in these settings - a hypothesis consistent with the experiences of teachers and students in the studies by Padilla Rodriguez et al. and Khlaif et al.

\section{Conclusion}

In response to our call for this Special Issue, we received a high volume of submissions from around the world. Our final selection of published studies represent a diversity of geographic areas, educational sectors, and topics related to the COVID-19 crisis, including equity challenges, assessment strategies, interpersonal connection issues, professional development supports, and successful course conversion approaches, in both the K-12 and higher education sectors.

The interest in this topic is obvious given the global crisis, which continues to this date of publication. Given that we cannot contain all the high-quality work and useful "lessons learned" from the COVID-19 crisis within the pages of this issue, OLJ will continue to publish selected COVID-related articles in subsequent issues. In addition, the Online Learning Consortium also has plans to develop other publication opportunities related to the pandemic - for example, special editions of white papers for the OLC Research Center, conference papers at an OLC event, or other formats. Please watch for additional communications from the OLC regarding these potential opportunities. 